\title{
Simultaneous front and rear faces flash characterization method for thin cylindrical multilayered composites.
}

\author{
L. Duquesne ${ }^{(1)(*)}$, C. Lorrette ${ }^{(2)}$, C. Pradere ${ }^{(3)}$, G. L. Vignoles ${ }^{(4)}$ and J-C. Batsale $e^{(3)}$ \\ (1) CNRS, LCTS, UMR 5801, F-33600 France, \\ (2) CEA, DEN, SRMA, LTMEx, F-91191 Gif-sur-Yvette, France \\ (3) Univ. Bordeaux, LCTS, UMR 5801, F-33600 Pessac, France \\ (4) Univ. Bordeaux, I2M, UMR 5295, F-33400 Talence, France \\ ${ }^{(*)}$ Corresponding author: duquesne@lcts.u-bordeaux1.fr
}

\begin{abstract}
The flash method, known as the most used experimental technique to measure the thermal diffusivity of solids, has been adapted to cylindrical highly diffusive and heterogeneous multilayered samples. In order to overcome the heterogeneities issue and give a more accurate estimation of the sample thermal properties, the front face and rear face thermal responses have been simultaneously recorded over a large characteristic frequency range using an infrared camera. A homogeneous monolithic SiC cylinder has been tested to validate this experimental method which has finally been applied to $2 \mathrm{D}$ bi-layered $\mathrm{SiC}_{\mathrm{f}} / \mathrm{SiC}$ cylinders used for nuclear fuel cladding.
\end{abstract}

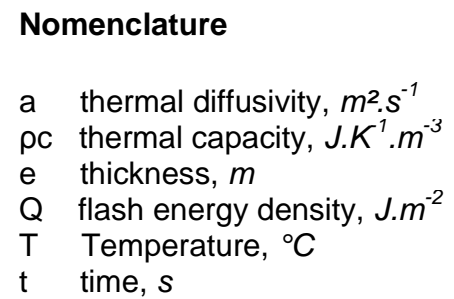

\section{Introduction}

The flash method was introduced by Parker et al. [1] and has become the most used experimental technique to measure the thermal diffusivity of solids. During the last decades, numerous estimation techniques based on the flash method have been proposed for the thermal characterization of flat samples [2]. Recent advances have led to the extension of the flash method to measure the thermal diffusivity of cylindrical homogeneous samples (lead foils). The validity of the analytical model based on quadrupole formalism and developed for flat samples has also been proved except for extremely curved samples [3]. This method allows following the thermal behaviour of a sample by a brief heating of the front surface of an opaque sample and observing the temperature evolution of the rear surface. Moreover, the different existing methods of thermal characterization use either front face or rear face signals obtained after a flash and lead to estimate the material thermal effusivity or diffusivity. In the case of highly diffusive and heterogeneous materials, the estimation accuracy is impaired.

In this study, we extend the flash method of characterization adapted to cylindrical homogeneous samples to cylindrical highly diffusive and heterogeneous multilayered samples. In order to overcome the heterogeneities issue and give a better estimation of thermal properties, this new experimental approach combining front and rear faces signals is introduced. It gives access to equivalent thermal diffusivities by a combined analysis of the front face and rear face thermal responses over a large characteristic frequency range. Flash measurements have been performed on multilayered tubes made of ceramic matrix composite $\left(\mathrm{SiC}_{f} / \mathrm{SiC}\right)$ layers. The objective consists in illustrating this new experimental approach and to confirm the validity of the analytical model based on quadrupole formalism and developed for flat samples.

First of all, a theoretical approach of the analytical solutions for flash method using the combination of front and rear face method is presented. Then, an experimental set-up implemented for this combination of flashes is validated on a homogeneous monolithic $\mathrm{SiC}$ cylinder. Finally, results on a $2 \mathrm{D}$ bi-layered $\mathrm{SiC} / \mathrm{SiC}$ cylinder are detailed and discussed. 


\section{Theoretical model}

The interpretative model is constructed using the quadrupole formalism [4,5] and yields a Fourier series solution of the heat equation for both faces. Front face (Eq. 1.) and rear face (Eq. 2.) analytical solutions for a heat pulse imposed on a homogeneous plan sample surface are:

$$
\left\{\begin{array}{l}
T(0, t)=\frac{Q}{\rho c e}\left[1+2 \sum_{n=1}^{\infty}(1)^{n} \exp \left(-\frac{n^{2} \pi^{2}}{e^{2}} a t\right)\right] \\
T(e, t)=\frac{Q}{\rho c e}\left[1+2 \sum_{n=1}^{\infty}(-1)^{n} \exp \left(-\frac{n^{2} \pi^{2}}{e^{2}} a t\right)\right]
\end{array}\right.
$$

Then, the sum (Eq. 3.) and the difference (Eq. 4.) of front and rear faces analytical solutions can be obtained:

$$
\left\{\begin{array}{l}
T(0, t)+T(e, t)=\frac{2 Q}{\rho c e}\left[1+2 \sum_{k=1}^{\infty} \exp \left(-\frac{4 k^{2} \pi^{2} a t^{2}}{e^{2}}\right)\right] \\
T(0, t)-T(e, t)=\frac{4 Q}{\rho c e} \sum_{k=0}^{\infty} \exp \left(-\frac{(2 k+1)^{2} \pi^{2} a t^{2}}{e^{2}}\right)
\end{array}\right.
$$

Their asymptotic development for long time behaviour ( $\left.\mathrm{t}>\mathrm{e}^{2} / \mathrm{a}\right)$ leads to the following equations:

$$
\left\{\begin{array}{l}
T(0, t)+T(e, t) \cong \frac{2 Q}{\rho c e} \\
T(0, t)-T(e, t) \cong \frac{4 Q}{\rho c e} \exp \left(-\frac{{ }^{2} \pi^{2} a t^{2}}{e^{2}}\right)
\end{array}\right.
$$

The long time behaviour of the front and rear faces signals sum (Eq. 5.) represents the front face response after a flash applied to a e/2-thick sample, with an adiabatic rear face. The long time behaviour of the difference (Eq. 6.) represents a front face response after a flash applied to a e/2-thick sample, with an isothermal rear face (imposed temperature $\mathrm{T}_{0}$ ). These analytical results are summarized in the following figure (Fig. 1.):

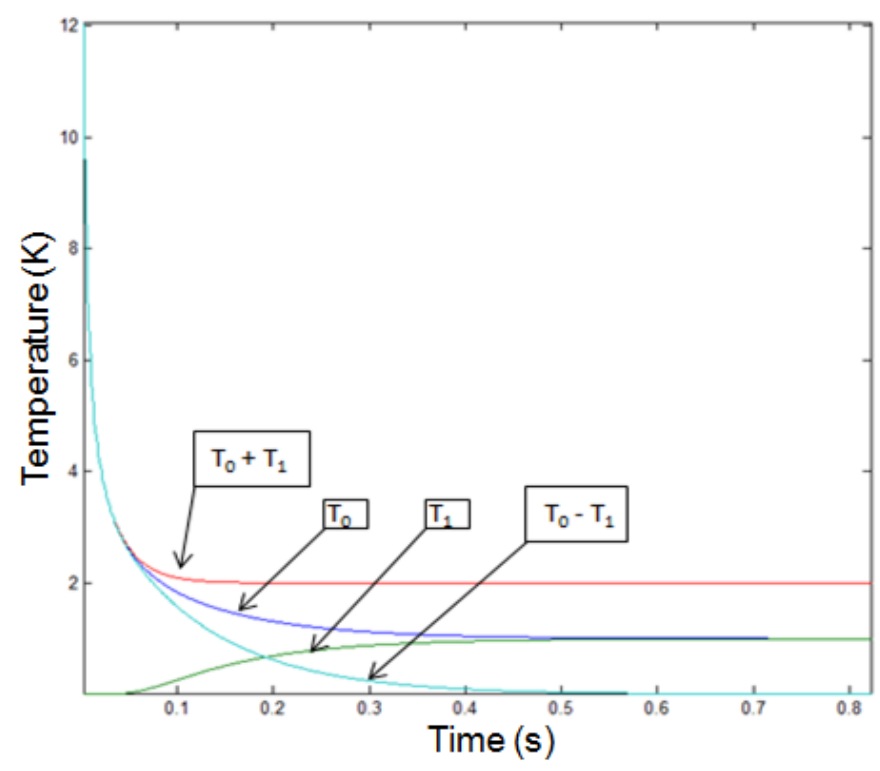

Fig. 1. Analytical responses for front (blue) and rear (green) face; sum (red) and difference (cyan) of these analytical responses (with $T_{0}=T(0, t)$ and $T_{e}=T(e, t)$ ) 


\section{Experimental approach}

\subsection{Experimental set-up allowing the combination of the front and rear faces signals}

A new experimental device has been developed in order to collect the front and rear face signals of a cylinder. The temperatures monitoring is provided by an InSb infrared camera (FLIR SC7000, detection window: 2.5 -5.5 $\mu \mathrm{m})$. Four external flash lamps are settled around the cylinder to ensure a quasi-uniform heating. Front face response is directly available by using a classical front face flash method (with a 1D transfer hypothesis). Contrariwise, in order to perform the rear face measurement, a mirror is set inside the tube. This mirror is settled with an angle of $45^{\circ}$, which allows recording the signals of the superior face of the tubular material. A gold mirror has been selected because it ensures a better reflection coefficient (it reflects over $98 \%$ in the infrared spectrum whereas a silver mirror only reflects $90 \%$ ). The direct influence of the heat pulse on the sensor is minimized by adding infrared filters (bandwidth: 315 to $710 \mu \mathrm{m}$ ). The temporal response of the flash is precisely measured using a photodiode (Thorlabs: PDA36A-EC) which allows catching the initial pulse heating instant. Moreover, the experimental device has been set up in a way to make the rotation between front and rear face position fast and user-friendly, as shown in Fig. 2. and Fig. 3.

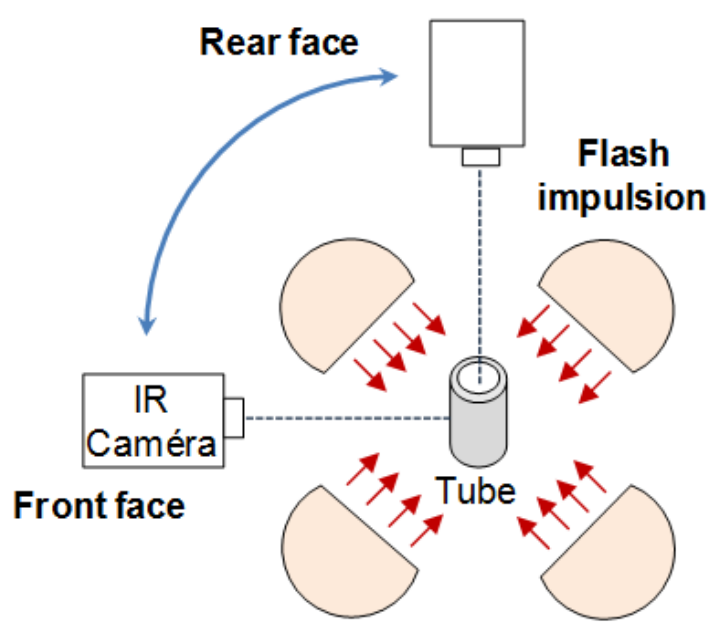

Fig. 2. Sketch of the experimental device for the combination of front and rear faces signals
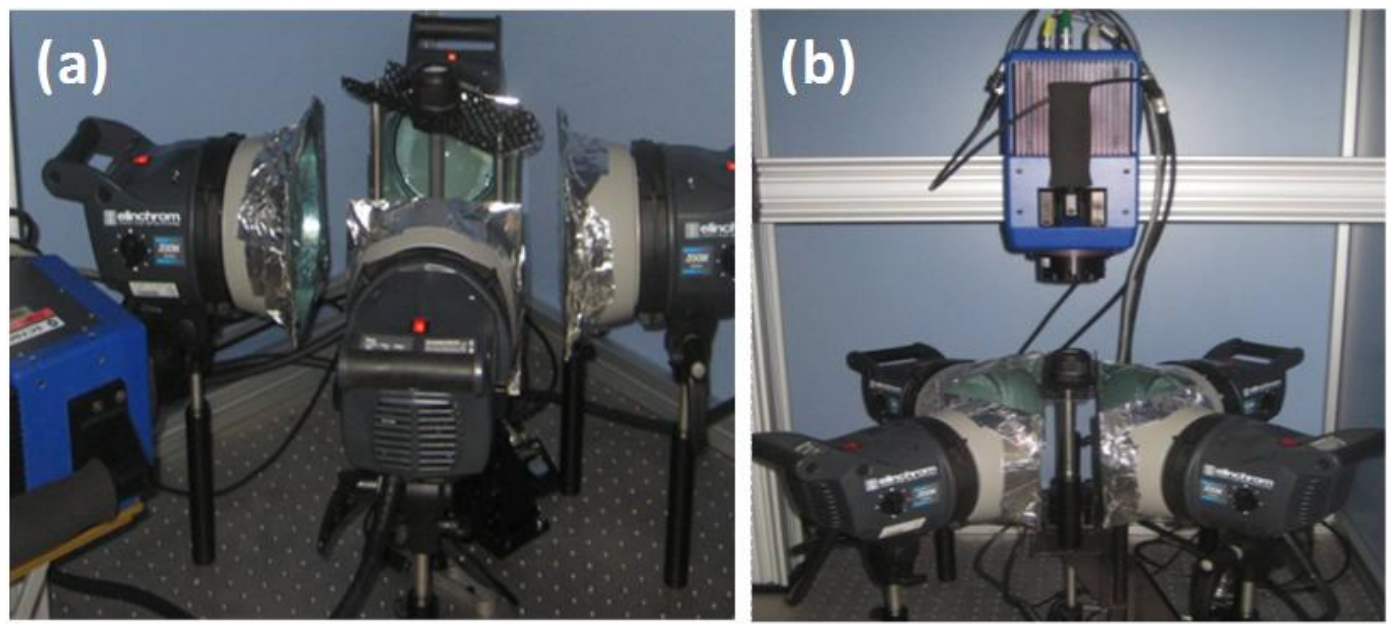

Fig. 3. Picture of the experimental set-up for (a) front face and (b) rear faces monitoring 


\subsection{Experimental protocol}

The front and rear faces thermal responses have been simultaneously recorded over a large characteristic frequency range using an infrared camera. Moreover, due to the high conductivity of our sample the influence of heat losses can be neglected. The sample has been illuminated by four flash lamps (Elinchrom: $300 \mathrm{~J}$ zoom action lamps - Digital 600RX power generators). Data have been recorded at 2500 frames per second. Only the mean of temperature on pixels at the center of the image has been recorded for the front and rear faces due to the curvature of the sample.

\section{Example of application}

This study focuses on developing measurement methods for thermophysical properties of multilayered tubes, made of composites $\left(\mathrm{SiC}_{\mathrm{f}} / \mathrm{SiC}\right)$, used for nuclear fuel cladding. For leak-tightness, mechanical and dimensional reasons, these tubes are highly heterogeneous. Global transverse and local variations of thermal properties of such a structure have to be identified, since they are essential to their final use - heat transfer in future nuclear power plants of next generation.

A validation of the experimental set-up for the combination of front and rear faces signals on a homogeneous "test" material is done. Then, results on 2D bi-layered heterogeneous $\mathrm{SiC}_{f} / \mathrm{SiC}$ are presented and discussed.

\subsection{Samples}

The samples are then carved to ensure that the difference between cylindrical and plane analytical thermal responses remains below 1\%. It means that e/rc<0.5 [3], where $r c$ is the inner radius of the outer layer and $e$ its thickness. In this case, Eq. 2 can be used.

The samples used for the validation of the method are tubular and made of a homogeneous monolithic SiC material (provenance: Boostec $\odot$ ). The samples to illustrate the proposed method are cylinders made of a 2D bilayered structure composed of ceramic matrix composite $\left(\mathrm{SiC}_{f} / \mathrm{SiC}\right)$ layers. They are prepared by braiding and filament winding techniques (provenance: CEA) and are about a millimeter thick.

\subsection{Validation on tubular homogeneous monolithic SiC material}

It has been proved that the residual of the sum and the difference of the signals is lower than the sum of the residual of the front and rear faces signals taken separately. Thereby, the reduced sensitivity to the thermal diffusivity is better using the sum and the difference of the signals than using both front and rear face signals only. Therefore, we assume that the diffusivity will be estimated with more accuracy.

The experimental front and rear face signals are obtained using the experimental set-up presented in section 3 (Fig. 2 \& 3). Their sum and their difference for a homogeneous monolithic SiC pipe are summarized in the following figure (Fig. 4):

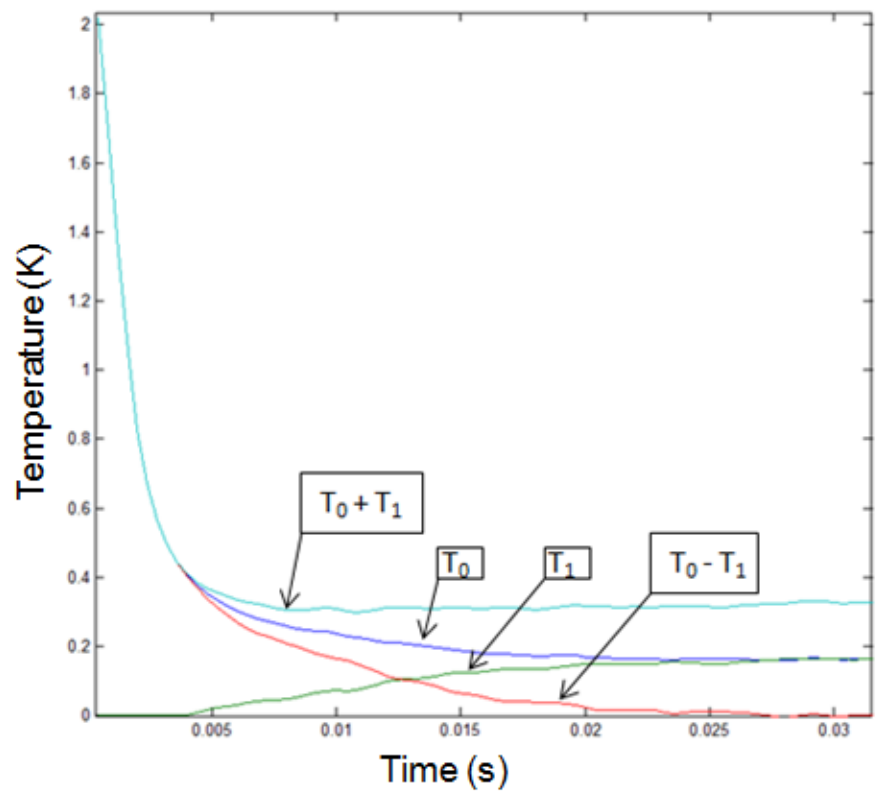

Fig. 4. Front (blue) and rear (green) faces signals; sum (cyan) and difference (red) of these signals 


\subsubsection{Study on the front and rear faces difference}

Regarding Eq. 6, we assume that when the material semi-infinite behaviour is over, the signal quickly describes a pure decreasing exponential response. Therefore, its logarithm is linear:

$$
\ln (T(0, t)-T(e, t))=\ln \left(\frac{4 Q}{\rho c e}\right)-a \frac{\pi^{2}}{e^{2}} t
$$

Thus, outside the semi-infinite environment, the signal can be easily minimized by a linear function with $a-a \frac{\pi^{2}}{e^{2}}$ slope. This linear regression leads directly to an estimation of the thermal diffusivity $a$, as it is explained in Fig. 5 .

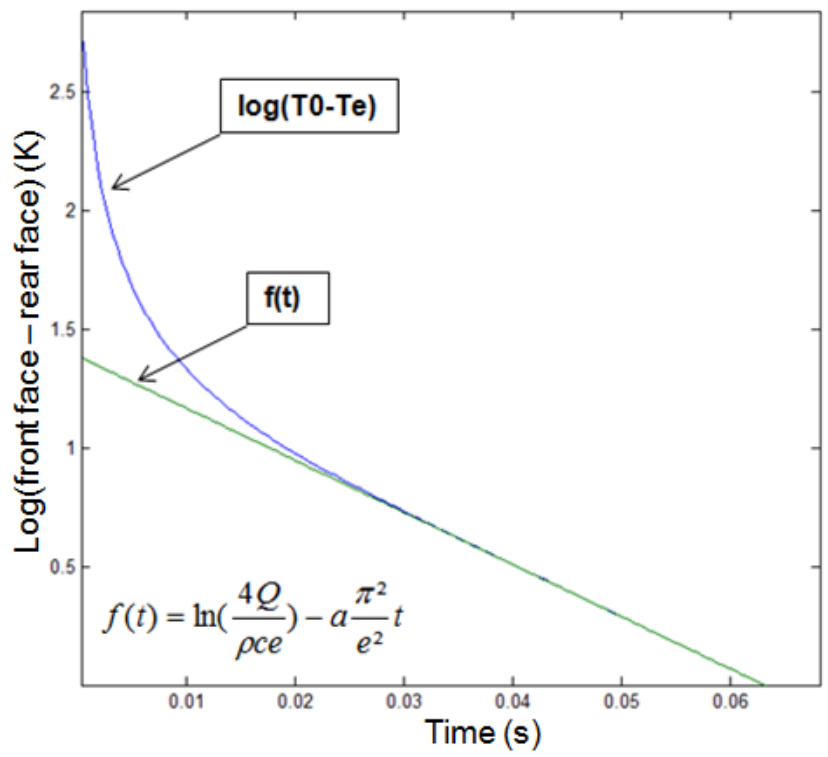

Fig. 5. Theoretical minimisation of the front and rear faces difference's logarithm

As a validation of this method, a homogeneous tubular monolithic SiC sample with a thickness of $2.1 \mathrm{~mm}$ is used (Boostec $\odot$ with a manufacturer data for thermal diffusivity of $70 \mathrm{~mm}^{2} / \mathrm{s}$ ). Theoretical expectations are experimentally reproduced on the test sample, as shown in Fig. 6, and the thermal diffusivity a is estimated with an error remaining inferior to $3 \%$.

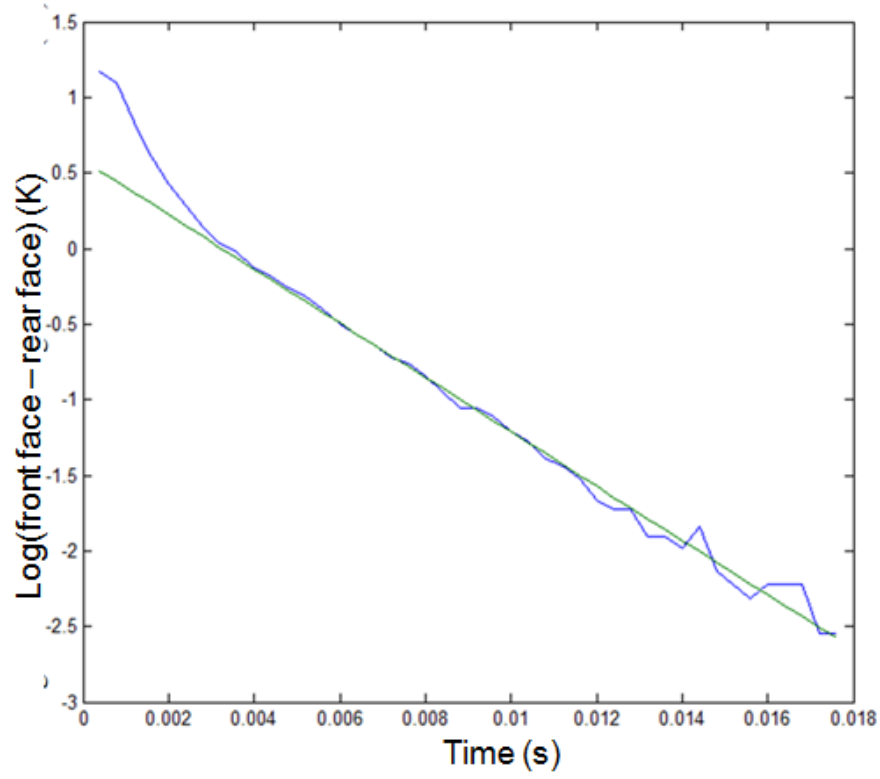

Fig. 6. Minimisation of the difference logarithm for a tubular homogeneous monolithic SiC sample 


\subsubsection{Study on the front and rear faces' sum}

Regarding Eq. 5, we assume that the signal describes the thermal response of a e/2-thick homogeneous sample, with a $\frac{2 Q}{\rho c e}$ long time adiabatic behaviour.

Theoretical expectations are also experimentally reproduced on the test sample and the thermal diffusivity $a$ is estimated (by a least square minimisation) with an error remaining inferior to $5 \%$.

\subsection{Experimental results for $2 \mathrm{D}$ bi-layered heterogeneous $\mathrm{SiC}_{\mathrm{f}} / \mathrm{SiC}$}

This new experimental approach is more sensitive than the ones based on the characterization either of the front face or of the rear face signals and the next step consists in applying it to a cylindrical bi-layered heterogeneous sample.

For a homogeneous material, it has been shown that the study of the combination of the signals corresponds to the study of a e/2-thick sample (Eq. $3 \& 4$ ). For the heterogeneous bi-layered sample, this combination allows the identification of the average of the apparent thermal diffusivities, separately identified with the front and rear face signals. In other words, this combination tends to homogenise our $\mathrm{SiC}_{f} / \mathrm{SiC}$ material. The experimental set-up is used in order to obtain front and rear faces thermal responses (Fig. 7.).

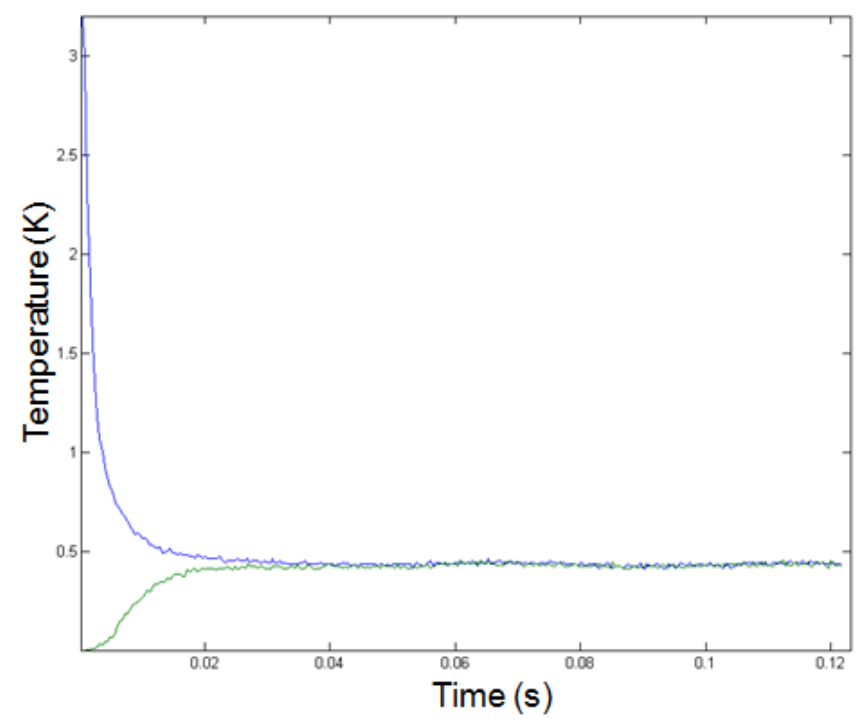

Fig. 7. Thermal response after a flash for front (blue) and rear (green) faces

Equation 3 allows the minimisation of the experimental sum of the thermal signals. Equation 7 is used for the minimisation of the experimental difference signals during intermediate times (Fig. 8.).

The thermal diffusivity is estimated at $11.4 \pm 0.5 \mathrm{~mm}^{2} / \mathrm{s}$ for the front face response and at $9.4 \pm 0.19 \mathrm{~mm}^{2} / \mathrm{s}$ for the rear face response. Thermal diffusivities are estimated at $9.7 \pm 0.6 \mathrm{~mm}^{2} / \mathrm{s}$ for the signals sum and at $10.3 \pm 0.15$ $\mathrm{mm}^{2} / \mathrm{s}$ for the signals difference. Using the signals sum and difference give the opportunity to estimate not two but four values of diffusivities. As expected, the sum and the difference thermal diffusivities estimations of the signals appear to act like the average of the front and rear faces estimations. 

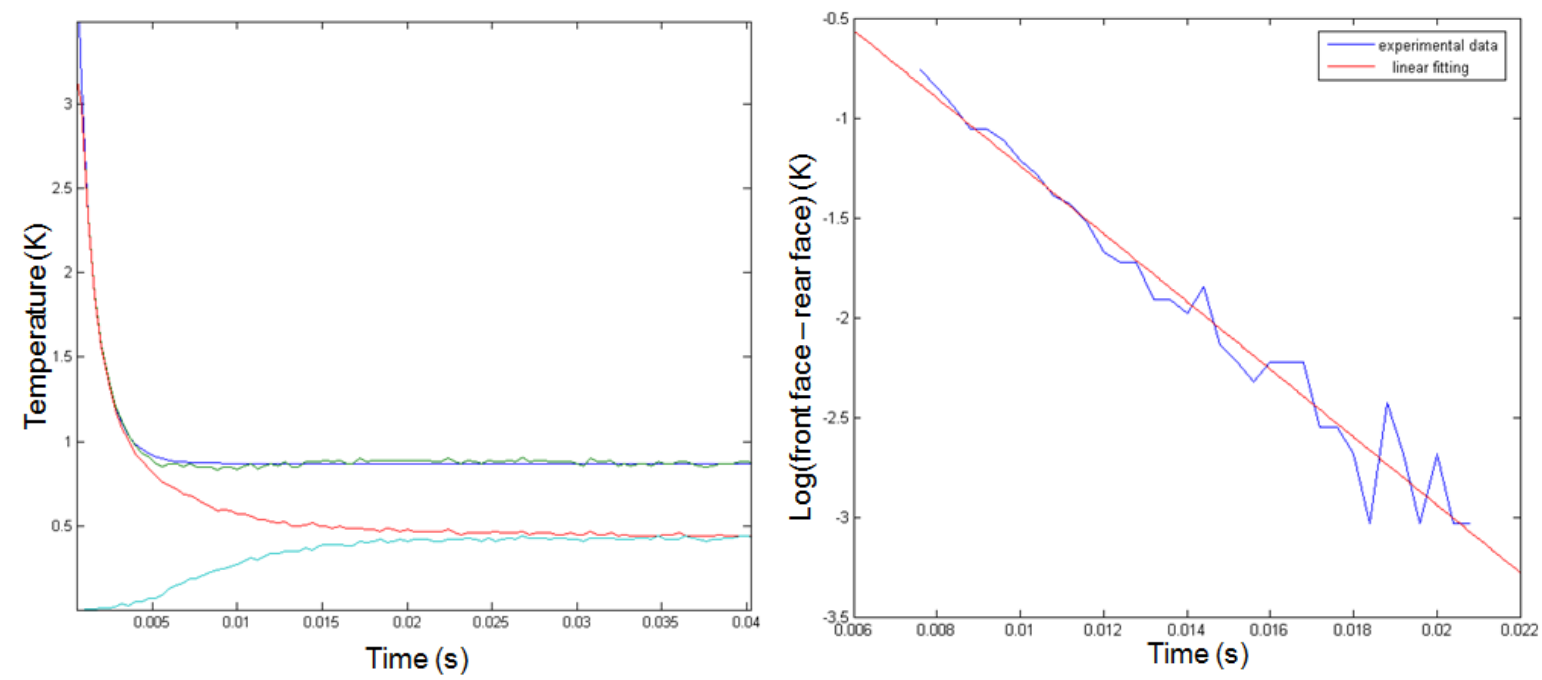

Fig. 8. Minimisation of the front and rear faces sum (left) and of the logarithm of the difference (right)

\section{Conclusion and Further works}

A method for the estimation of the radial thermal diffusivity for multi-layered tubular samples based on the combination of front and rear faces flash signals has been settled up. An experimental set-up has been developed and validated using a homogeneous monolithic tubular SiC test sample. Finally, this new approach allows estimating not two but four estimations of the thermal diffusivity of a bi-layered $\mathrm{SiC}_{\mathrm{f}} / \mathrm{SiC}$ real sample and provides results consistent with literature [6]. The developed experimental set-up tends also to homogenise the studied material and seems to be a suitable solution for the study of heterogeneous tubular materials.

\section{REFERENCES}

[1] W. J. Parker, R. J. Jenkins, C. P. Butler and G. L. Abbott, Flash Method of Determining Thermal Diffusivity, Heat Capacity, and Thermal Conductivity, Journal of Applied Physics, 32,1679, 1961.

[2] D.Balageas, Thickness or diffusivity measurements from front-face flash experiments using the TSR (thermographic signal reconstruction) approach, QIRT 2010-011.

[3] A.Salazar, R.Fuente, E.Apiñaniz, A.Mendioroz. Thermal diffusivity of nonflat plates using flash method. Review of Scientific Instruments. 82, 014902; doi: 10.1063/1.3529431, 2011.

[4] H.Stehfest, Numerical inversion of Laplace transforms. Communication of the ACM, 13, 10, 624, 1970.

[5] J-C.Batsale, D.Maillet, A.Degiovanni, S.André, C.Moyne, Thermal Quadrupoles: Solving the Heat Equation through Integral Transforms. Wiley, Chichester, 2000.

[6] R. Yamada, N. Igawa, T. Taguchi, S. Jitsukawa, Highly thermal conductive, sintered SiC fiber-reinforced 3D$\mathrm{SiC} / \mathrm{SiC}$ composites: experiments and finite-element analysis of the thermal diffusivity/conductivity, J. Nucl. Mater. 307-311, 1215-1220, 2002. 